[Vicino Oriente XVII (2013), pp. 165-173]

\title{
LO STILE ‘POETICO’ DELLE HISTORIOLAE ITTITE
}

\author{
Rita Francia - Sapienza Università di Roma
}

The language of Hittite Historiolae and magic spells sometimes displays a stylistic structure that can be defined as 'poetic'. The style of these compositions is similar to that of 'rhythmic prose', according to the definition of E. Norden.

Keywords: historiolae; rituali magici; poesia; prosa ritmata; ittita

I rituali magici ritrovati nei depositi documentari di Hattuša rappresentano una parte numericamente rilevante dell'intero corpus ittita, costituendo una testimonianza importante per la ricostruzione dell'aspetto culturale di questo popolo' ${ }^{1}$.

Lo studio dello stile del linguaggio dei rituali ittiti ha portato alla luce delle caratteristiche per noi inaspettate ${ }^{2}$. Già in lavori precedenti abbiamo posto l'attenzione sulle

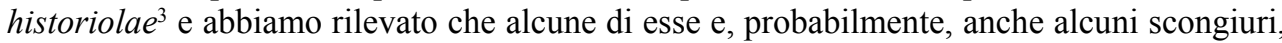
presentano delle particolarità linguistiche e stilistiche tali da potersi definire 'fuori dalla norma', avendovi individuato un andamento cadenzato e ritmato; riteniamo che la definizione più adatta per definire lo stile di questo linguaggio sia "poetico'4. La complessità di queste composizioni non può essere frutto di improvvisazione o casualità: essa rivela l'esistenza di una tradizione ben fondata che, se da un lato affonda le sue radici nella cultura indoeuropea d'origine, dall'altro ha certamente subito gli influssi delle altre popolazioni, indoeuropee e non, con cui gli Ittiti si sono trovati ad avere a che fare, come i Pala, i Hatti, antichi abitanti dell'Anatolia, e i Hurriti, ma anche quelle di ambito mesopotamico e nord siriano con cui gli Ittiti ebbero stretti contatti. Il risultato è una poesia semplice, sia nella forma che nei contenuti, come si addice a componimenti di matrice popolare, con un andamento simile alle nostre filastrocche, con una ricchezza e una varietà di forme espressive tipiche dei testi destinati ad essere tramandati oralmente. Le historiolae, infatti, analogamente ai rituali in cui sono incardinate, sono il prodotto della cultura volgare dell'epoca: composte in ambito popolare per una fruizione popolare, il cui mezzo di trasmissione era l'oralità. Questi componimenti sono estremamente significativi, riguardo al contenuto, perché riflesso di un ambito culturale di cui non restano molte testimonianze,

1 Stando a $\mathrm{HW}^{2}$ Lief. 2 (1977), p. 85 i rituali rappresentano il 70\% dell'intera documentazione. Per le sigle e le abbreviazioni usate rimandiamo a H. G. Güterbock - H. A. Hoffner, The Hittite Dictionary of the Oriental Institute of the University of Chicago. L-N (Chicago 1989), pp. XV-XXVIII.

2 Il presente contributo è parte di uno studio più ampio che stiamo conducendo sulle historiolae e sugli scongiuri nei rituali ittiti.

3 Definiamo le historiolae ittite narrazioni brevi di argomento non necessariamente mitologico, ambientate in un tempo passato o presente, i cui protagonisti sono esseri umani, divini, animali o piante, inserite in incantesimi il cui scopo è di fungere, esplicitamente o implicitamente, da paradigma per le azioni rituali di cui fanno parte. Per una definizione generale di historiola si veda Frankfurter 1993, 458, con bibliografia precedente.

4 Francia 2004, a tal proposito si vedano anche le annotazioni di Melchert 2007, e il nostro "Storia degli studi sulla poesia ittita e una nuova chiave di lettura di un testo 'classico': CTH 414”, (2013). 
e 'corali' perché espressione stratificata di una cultura che si è protratta nel tempo5. I testi nella forma in cui ci sono giunti sono, probabilmente, solo una delle numerose fogge in cui ognuno di essi circolava: quella che, o per casualità o perché ritenuta particolarmente significativa, fu raccolta e messa per iscritto, destinandola così alla conservazione.

L'uso del termine 'poesia' può sembrare inappropriato per la documentazione a cui ci riferiamo: poesia evoca ben altre aspettative in noi lettori moderni; riteniamo, pertanto, di dover precisare cosa intendiamo con esso e, soprattutto, quali significative caratteristiche abbiamo rilevato nei testi esaminati che ci hanno indotto a farvi ricorso.

Il Dizionario della Lingua Italiana Treccani on-line attribuisce a 'poesia' una serie di significati, quelli che a noi interessano sono due:

1.a. «L'arte (intesa come abilità e capacità) di produrre composizioni verbali in versi, cioè secondo determinate leggi metriche, o secondo altri tipi di restrizione; con un certo grado di approssimazione si può dire che il sign. di poesia è individuabile nell'uso corrente e tradizionale nella sua contrapposizione a prosa, in quanto i due termini implicano rispettivamente e principalmente la presenza o l'assenza di una restrizione metrica»;

2.a. «Il carattere di opere o parti di opere ritenute particolarmente ispirate e suggestive: [...]. In questa accezione, tratto pertinente non è più il metro o un equivalente del metro, ma l'elevatezza concettuale e formale, al di là di una rigida adesione a schemi formali di metro, ritmo, struttura $[\ldots] \gg$.

La prima definizione (1.a) considera essenzialmente l'aspetto formale del testo: la presenza di uno schema metrico di base in una composizione la contrappone alla prosa, quindi un componimento è poetico se rispetta una struttura metrica; la seconda (2.a) invece fa riferimento ad aspetti intrinseci, come «l'elevatezza concettuale e formale».

Nel linguaggio letterario, vengono definite poetiche quelle opere che utilizzano quanto più possibile gli artifici della 'funzione poetica', intesa in senso jakobsoniano ${ }^{6}$. In esse si sfruttano al sommo grado le proprietà musicali e ritmiche della lingua e, nel contempo, si fa uso del codice linguistico violando le regole della comunicazione quotidiana, per conseguire una maggiore intensità espressiva. In queste opere inoltre si ricorre sovente all'uso di figure retoriche per creare immagini nuove e suggestive tali da colpire l'uditorio. Si può parlare dunque di poesia anche per scritti, testi letterari e non, che di fatto 'poesie' (cioè componimenti composti ricorrendo ad artifici metrici) non sono, ma che presentano un particolare linguaggio la cui funzione predominante è quella 'poetica'. Lo scopo principale di questa funzione è 'far poesia', arricchire cioè il messaggio di significati in più che prescindono dalla sfera semantica ed attengono alla sua tessitura fonico-ritmica. Là dove predomina la funzione poetica, il significato della comunicazione è affidato essenzialmente al modo in cui il messaggio è costruito. Le parole pertanto sono importanti non solo e non tanto per i contenuti di cui sono portatrici, ma per il modo in cui vengono

Melchert 2007

6 Jakobson 1966, 181-218. Con il termine 'funzione' riferito al linguaggio intendiamo il modo in cui esso viene adoperato relativamente allo scopo per cui il messaggio espresso è formulato. Pertanto, lo 'scopo' è di fondamentale importanza nella produzione del messaggio: è anteriore ad esso e incarna l'intenzione sottesa al messaggio stesso. La 'funzione' è, in ultima analisi, la forma che il messaggio assume una volta che è stato realizzato. 
organizzate nel testo e per le sensazioni e le informazioni aggiuntive che possono trasmettere con il loro suono. In questi messaggi l'emittente, cioè colui che invia il messaggio, sfrutta le componenti ritmiche e musicali della lingua, utilizzando il codice linguistico in modo arbitrario, violando cioè le norme della comunicazione quotidiana, per ottenere una maggiore intensità espressiva e arricchire il messaggio di significati più profondi, cioè di altre connotazioni altrimenti assenti.

Questa funzione, in epoca contemporanea, può essere ritrovata sia in opere letterarie, sia in testi che letterari non sono, come, ad esempio, gli slogan pubblicitari ${ }^{7}$.

\section{LA FILASTROCCA}

Un altro genere testuale in cui si rilevano caratteristiche 'poetiche', almeno nell'aspetto formale (per la presenza di metro, ritmo e rima) è la 'filastrocca'. E' questo un componimento di matrice popolare presente in tutte le culture, caratterizzato da particolarità linguistiche e formali specifiche, diverse da qualunque altro tipo di componimento. Dare una definizione della filastrocca non è semplice, per tracciarne le caratteristiche esaminiamo le principali che ne sono state date. Il Dizionario della Lingua Italiana Treccani on-line, definisce la 'filastrocca' una «canzonetta o composizione cadenzata (talvolta anche in forma di dialogo), generalmente in metri brevi, assonanzati o ritmati, con ritmo celere, formata di frasi collegate tra loro da richiami meramente verbali, che viene recitata o cantata dai bambini nei loro giochi, o anche dagli adulti per divertire, quietare, addormentare i bambini stessi»».

L'etnolinguista Glauco Sanga ${ }^{8}$, esperto di letteratura folklorica, a proposito della filastrocca scrive: «Il genere della filastrocca è oscurissimo, tanto da non essere delimitabile come genere se non all'ingrosso e con continue incertezze. Una definizione formale è assai difficile, perché entra continuamente in contatto con i generi contermini: la filastrocca viene eseguita con una recitazione ritmata, ma può essere anche cantata; non ha caratteri formali tali che la possano distinguere dal gioco infantile e dalla conta, o dalla preghiera, o dallo scongiuro. Sul piano semantico, sembra alla nostra coscienza che la filastrocca si caratterizzi solo in negativo, come testo nonsense. Questa impressione è del tutto fallace, e sottolinea solo la distanza culturale tra noi e i testi di letteratura popolare che non comprendiamo più; d'altra parte è difficile da rimuovere finché non si propone una qualche credibile ipotesi di lettura».

7 Un testo letterario in prosa in cui è facilmente individuabile la prevalenza della funzione poetica è senza dubbio l'incipit del romanzo manzoniano I promessi Sposi: «Quel ramo del lago di Como, che volge a mezzogiorno, tra due catene non interrotte di monti, tutto a seni e a golfi, a seconda dello sporgere e del rientrare di quelli, ...». Evidente è la differenza tra questa descrizione e quella che, ad esempio, si trova in una guida del Touring Club Italiano pur riferita agli stessi luoghi: «Magnifico bacino prealpino, totalmente lombardo ... È chiamato anche Lario. Le sue acque sono all'altitudine di $199 \mathrm{~m}$; la superficie è di $146 \mathrm{~km}^{2}$, la lunghezza di circa $50 \mathrm{~km}$, la larghezza massima di $4,4 \mathrm{~km}$, la profondità di $410 \mathrm{~m}$. ...» (Guida rapida d'Italia. Vol. I. Touring Club Italiano 1992). Al linguaggio pubblicitario appartiene lo slogan di un noto whisky: «il cuore della notte batte al ritmo di Ballantine's», macroscopica metafora, arricchita da allitterazioni, (notte, batte) e assonanze (cuore, notte).

8 Goi 1991, Introduzione. 
Secondo il linguista Mario Alinei甲 la filastrocca è: un «Componimento in versi brevi, con ripetizioni di sillabe e parole"; lo studioso ritiene che la migliore definizione del genere sia stata data da Anselmo Roveda: «la filastrocca è [...] un testo di poesia popolare, prodotto o conosciuto dai bambini di una data cultura popolare, legato al mondo infantile della cultura popolare di riferimento, cultura alla quale si informa nei contenuti e nei valori, anche inconsci e di sostrato, usato dai bambini o con loro, dagli adulti, con funzioni (insegnamento, intrattenimento,...) e in generi (ninna nanna, conte,...) vari; nonostante la varietà di generi e funzioni, è in definitiva: un testo, per lo più breve, d'uso o destinazione infantile, con spiccate connotazioni ritmiche» ${ }^{10}$. A questa definizione di testo Alinei aggiunge anche gli aggettivi «recitato o cantato/cantilenato» ${ }^{11}$.

Da queste definizioni si evince che le principali caratteristiche della filastrocca sono: essere recitata o cantata/cantilenata, avere una spiccata componente ritmica, essere composta in versi brevi, mostrare ricchezza di assonanze e di collegamenti lessicali tra le singole frasi che la compongono, dunque ricca di molte ripetizioni ed essere destinata ad un pubblico infantile. La definizione di Sanga evidenzia che le sue caratteristiche formali non la distinguono, tra l'altro, dallo scongiuro, parere questo condiviso anche da Alinei ${ }^{12}$ : $" \mathrm{Ci}$ sono poi altri etnotesti formalmente avvicinabili alle filastrocche, ma distinti da essi in quanto di solito riservati agli adulti, come gli scongiuri e le formule di incantesimo o magiche (magic spells, charms).... Come tutti sanno, ve ne sono poi innumerevoli altre, con o senza figure magiche o religiose, che guariscono il singhiozzo, i crampi, allontanano i fantasmi, fanno venire bene il burro, proteggono dal malocchio....».

Sia gli scongiuri che le filastrocche dunque custodiscono uno spaccato di cultura popolare, di tradizione orale, affidata solo in un secondo tempo alla scrittura, mantenendo intatte alcune caratteristiche loro peculiari tra cui l'orecchiabilità data dal ritmo semplice ma efficace.

Naturalmente questo tipo di linguaggio si serve degli artifici tecnici che riconosciamo anche nella poesia ma è ben lontano dalla 'poesia' vera e propria. Si tratta infatti di etnotesti, cioè di «testi orali che rappresentano un'espressione autonoma della cultura di una comunità linguistica: [...]. Testi fissati dalla tradizione (proverbi, indovinelli, preghiere, ecc.) o testi solo parzialmente formalizzati (leggende, storie, etc. $)^{13} \gg$.

Gli etnotesti mostrano particolarità formali che li accomunano a quelli poetici nel senso proprio del termine: innanzitutto gli espedienti tecnici del linguaggio con cui sono composti, che non si ritrovano nel comune parlare quotidiano, come le figure del significante (assonanze, consonanze, allitterazioni, rime etc.).

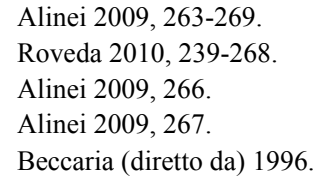




\section{LA PROSA RITMATA}

Caratteristiche analoghe a queste sono quelle individuate da Eduard Norden nel suo celebre studio sulla prosa d'arte antica del 1898, ma ancor oggi attuale, in quel tipo di prosa da lui etichettato «prosa d'arte»e, più specificamente «prosa ritmata» e «prosa poetica». La 'poesia' è una creazione moderna: la distinzione tra 'prosa' e 'poesia' era del tutto estranea alla mentalità arcaica: Isocrate afferma che «la prosa non deve essere mai metrica, ma deve essere sempre ritmica ${ }^{14}$ ponendo così una netta distinzione tra una composizione in metro e una ritmata. Nell'analizzare la prosa poetica, Norden ${ }^{15}$ osserva che alcune tipologie testuali, tra cui le formule magiche, sono concepite in una lingua diversa dal quotidiano, in una prosa che si differenzia dalla lingua comune ed è ben più antica della poesia. Le caratteristiche che pongono questa prosa su un piano diverso rispetto a quella del parlare comune sono: la solennità, e infatti essa è realizzata in forma ritmata «non uguale al canto, ma ad esso affine (recitativo)», e il ricorso ad alcuni espedienti fonici, come l'allitterazione e la rima, al fine di elevare il discorso e, contemporaneamente, anche far sì che esso si imprima nella memoria degli uditori. In una società in cui il mezzo di trasmissione primario è quello orale, il tenere a mente quanto si è udito è di somma importanza.

\section{IL CARMEN LATINO}

Restando in ambito classico, manifestazioni di questo tipo di prosa si hanno anche nella documentazione latina risalente alla fase cosiddetta preletteraria. A Roma per soddisfare l'esigenza di realizzare un tipo di espressione elevata, distinta cioè dalla comunicazione quotidiana, si ricorreva al carmen. Con questo termine si indicava in modo indistinto ciò che solo in un secondo momento si qualificò come prosa da una parte e poesia dall'altra ${ }^{16}$. Il carmen era espresso con un discorso ritmato, paragonabile ad una poesia fortemente formulare (concepta verba), ma senza schemi fissi, come quelli del verso (conformemente alla definizione di Isocrate). Questo tipo di espressione può anche definirsi 'prosa commatica', modulata e ritmata, arricchita da armoniose cadenze. La fase preletteraria latina è definita da De $\mathrm{Meo}^{17}$ : «Un lungo periodo, durante il quale alla parola pronunciata o scritta in particolari modi e circostanze si attribuì forza magica capace di determinare fatti ed eventi, sicché bastava nominare le potenze misteriose e invisibili per assicurarsene l'intervento, o pronunciare la maledizione perché il destinatario ne fosse colpito, a meno che altre forze, anch'esse invocate, non avessero agito in senso contrario (il fascinus verborum, l'influenza maligna, per essere rimosso o annullato esigeva atti e formule apotropaiche: fascinum arcere). E tale era l'efficacia del carmen da non risultare vanificata neppure dalla mancata comprensione delle parole da parte di chi le pronunciava o di colui al quale erano dirette, ché esse agivano anche in forza del loro mistero.».

Nella Roma arcaica con il carmen erano espresse tutte quelle manifestazioni letterarie formulate con un linguaggio diverso da quello comune, proprio del quotidiano, solenne e tale da essere ricordato. In questa forma furono dunque composti sortilegi, incantesimi,

14 art. fr. 12 (Baiter - Sauppe 1839).

15 Norden 1898, vol. I, 40

16 De Meo 1986, 139.

17 De Meo 1986, 139. 
formule di scongiuro, malefici, ma anche proverbi, ricette mediche, filastrocche, canti militari, preghiere e suppliche agli dei, nonché leggi.

La caratteristica principale del carmen era di essere composto secondo un andamento cadenzato, ritmato, scandito, oltre a mostrare figure retoriche come il parallelismo tra membri di frase, l'antitesi, la ripetizione, l'assonanza, la figura etimologica, l'omoteleuto e l'allitterazione, figura tipica della letteratura latina. Il ritmo, l'intensificazione semantica, la ripetitività formulare erano tipiche del linguaggio popolare solenne e, paradossalmente, anche di quello ad esso per certi versi opposto, il giuridico-sacrale ${ }^{18}$. Il comune denominatore di questi generi letterari è di doversi imprimere nella memoria degli ascoltatori, di essere ricordati e tràditi oralmente, da qui la necessità di questi artifici retorici.

\section{HISTORIOLAE ITTITE E PROSA RITMATA}

In uno studio sui testi liturgici e sulle preghiere latine e umbre, Calvert Watkins riprende il concetto di 'prosa ritmata' ('rhythmic prose') di Norden, riferendosi ad una prosa che non può né essere ricondotta né a quella normale (frasi lunghe, sintatticamente complesse) né alla poesia con struttura metrica. A conclusione delle sue riflessioni, lo studioso americano sottolinea la stretta familiarità individuabile tra la 'prosa ritmata' e la struttura poetica in versi: "The traditional label is "rhythmic prose", but that is far from being even observationally adequate, the more so since the typical responsion of equivalence tokens that "rhythmic prose" shows is precisely "the essencial characteristic of verse'» ${ }^{19}$.

Ritornando ai testi ittiti di contenuto rituale, non si può fare a meno di notare come alcune historiolae e scongiuri siano stati elaborati ricorrendo ad espedienti retorici ricercati, tanto da non poter essere posti sullo stesso piano della normale espressione in prosa in cui sono concepite altre sezioni di questi stessi testi. Un esempio di questa particolare espressione linguistica è dato dal brano seguente desunto dal rituale "del Signore della lingua" (EME-aš EN-aš) KUB 12.62 + KBo 53.3 (CTH 338):

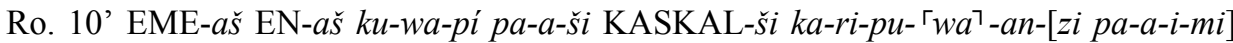
(11') UR.MAH tar-wa-u-wa-an-zi pa-a-i-mi a-li-li war- $\left\ulcorner^{\swarrow} u^{\top}{ }^{-} w a-a n-z i\right.$ pa $\left\ulcorner a-i-m i i^{\urcorner}\right.$(12') DUMU.<LÚ.> $\mathrm{U}_{19}{ }^{\mathrm{LU}}$ la-la-u-wa-an-zi pa-a-i-mi KASKAL-aš-za ka-ri-pu-wa-an-zi Ú-UL (13') me-em-ma-i UR.MAH-aš-za tar-wa-u-wa-an-zi UL me-em-ma-i a-li-la-aš-za (14') war-šu-wa-an-zi Ú-UL me-em-ma-i ${ }^{\mathrm{d}} \mathrm{UTU}-u \check{s}-z a$ HUL-mu-uš $\mathrm{EME}^{\mathrm{HI} . \mathrm{A}}$ (15') UL me-<em>$m a-i /$

Ro. 10' 'Signore della lingua, dove vai?' 'vado alla strada per <la sua capacità di> divorare <lo spazio $>20$, (11') vado dal leone per $<$ la sua capacità di $>$ balzare, vado dall'uccello alili $^{21}$ per $<$ la sua capacità di $>$ beccare, (12) vado dall'essere umano per $<$ la sua capacità di $>$ parlare"'. La strada non (13') si rifiuterà di divorare $<$ lo spazio $>$, il leone non

18 De Meo 1986, 154.

9 Watkins 1995, 231

20 Così Archi 1998.

21 Si tratta di un uccello impiegato nell'ambito dell'ornitomanzia: HED, vol. I, p. 34 e sg.; HW², Lfg. 1, p. 59. 
si rifiuterà di balzare, l'uccello $a$. (14') non si rifiuterà di beccare, la divinità solare non rifiuterà le malvagie lingue.

Analisi stilistica ${ }^{22}$ :

A (10’) lalaš išḥaš kuwapi pāšsi

B palši karipuwan[zi pāimi $]$

$\mathrm{C}{ }^{*}$ walkwi $^{23}$ tarwauwanzi paimi

D alili war $\ulcorner\check{s} u\urcorner$ wanzi $p\ulcorner\bar{a} i m i\urcorner$

E (12') antuhšsi lalauwanzi pāimi

F palšaš=za karipuwanzi natta memmai

$\mathrm{G}$ *walkuwaš=za tarwauwanzi natta memmai

$\mathrm{H}$ alila-aš $=z a(14 ')$ waršuwanzi natta memmai

I $\mathrm{d}$ Ištanuš =za idalamuš lalaš natta memmai /

Il brano è composto in stile dialogico drammatico 'botta e risposta'. Il verso (A) è di apertura e introduce il dialogo con la domanda: "Signore della lingua, dove vai?", a cui seguono due strofe di quattro versi ognuna, di cui la prima (B-E) costituisce la prima parte del dialogo e la seconda (F-I) la ripresa enfatica di esso da parte dell'interlocutore. Riteniamo di individuare in queste due strofe la costruzione formulare litotica (argomento negazione del contro argomento) la cui funzione è di conferire maggiore enfasi all'argomento $\operatorname{stesso}^{24}$. Infatti nella prima strofa si susseguono delle frasi affermative, mentre la seconda è costituita da frasi negative che però rendono positivo il significato del verbo di frase: memmai "rifiuterà", ma natta memmai "non rifiuterà", cioè "accetterà".

I versi sono isocoli, ripetono cioè la stessa struttura sintattica, conferendo al brano un andamento ripetitivo a blocchi distinti: in B-E si ripete la costruzione dat. loc. - acc. (verbo all'infinito) - verbo:
B KASKAL-ši karipuwan $[z i \quad$ paimi $]$
dat.loc. - acc. (infinito) - verbo
C *walkwi tarwauwanzi paimi
dat.loc. - acc. (infinito) - verbo
D alili war $\ulcorner\check{s} u\urcorner$ wanzi $\quad p\ulcorner a i m i\urcorner$ dat.loc. - acc. (infinito) - verbo
E antuḩsi lalauwanzi paimi. dat.loc. - acc. (infinito) - verbo

In F-I invece ricorre la costruzione soggetto (per cui è specificata la desinenza $-a \grave{s}$ ) $+z a$ acc. - negazione - memmai:

F soggetto $+z a$ - acc. (infinito) - negazione - verbo

22 Per l'analisi stilistica non si è tenuto conto della lunghezza delle righe ma delle singole frasi, in strutture che chiamiamo 'versi', con i logogrammi sostituiti dalla corrispondente parola ittita.

23 Per la lettura del sumerogramma UR.MAH = *walkuwa-: Weeden 2011, 164.

24 Watkins 1995, 686. 


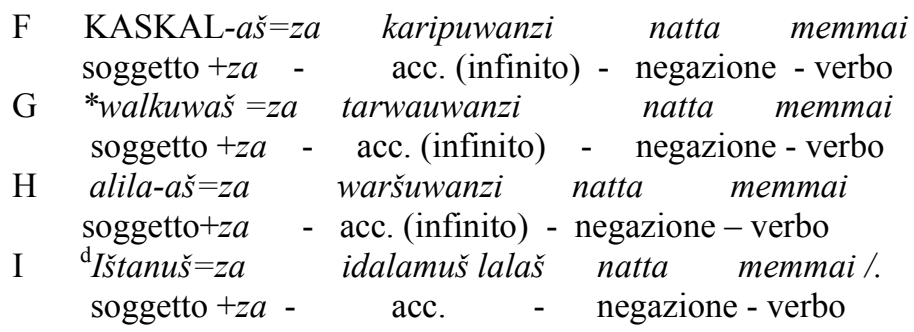

In entrambe le strofe si individua l'omoteleuto: nella prima strofa per la ripetizione del verbo finale paimi, mentre nella seconda del verbo memmai; i versi di ciascuna strofa sono isocoli, avendo uguale numero di parole e stessa costruzione ${ }^{25}$. Nei versi (B)-(E) e in (F)(H) c'è anche una rima interna data dalla desinenza dell'infinito - uwanzi che ricorre sempre nella stessa posizione, al centro del verso; interessante è anche osservare che i primi tre versi delle strofe iniziano tutte con una sillaba che contiene il suono AL: pALšsi, *wALkwi, ALili - pALša ̌̌, *wALkuwaš, ALilaš generando un legame omofonico tra i versi. In (C) e in (G) vi è l'allitterazione per la ripetizione di WA ( *walkwi tarwauwanzi - *walkuwaš $=z a$ tarwauwanzi)

\section{CONCLUSIONI}

I rituali possono ritenersi a buon titolo uno spaccato di letteratura popolare, ed è proprio in questo la loro peculiarità: nella loro genesi e formazione è da ricercare la spiegazione della loro tipologia formale e stilistica, come già considerato per le medesime espressioni testuali di ambito classico. L'accuratezza e le particolarità stilistiche che abbiamo riscontrato nel brano analizzato sono del tutto simili a quelle della prosa ritmata e della prosa poetica. Il tipo di 'poesia' è paragonabile a quella semplice e popolare che si ritrova nelle filastrocche, negli scongiuri, nelle formule magiche delle letterature classiche e non. Per queste ragioni riteniamo che si possa usare a buon titolo il termine 'poetico' nel riferirsi allo stile di questo genere testuale, intendendo con ciò quella particolare poetica propria della 'prosa d'arte antica', cioè la prosa poetica e la prosa ritmata. Infatti lo stile in cui le historiolae ed anche alcuni scongiuri sono scritti si discosta dalla semplice prosa, ma nel contempo non può definirsi a pieno 'poetico'. In questo i testi ittiti non sono diversi dalle composizioni arcaiche e popolari analoghe proprie di altre culture, mostrando una prosa «non uguale al canto, ma ed essa affine», secondo la definizione di Norden.

25 Riteniamo il sintagma aggettivo - nome (idalamuš lalaš) in (I) una unità, conformemente a Kloekhorst 2011. 


\section{BIBLIOGRAFIA}

ALINEI, M.

2009 Le origini linguistiche e antropologiche della filastrocca: Quaderni di Semantica XXX/2 (2009), pp. 263-290.

ARCHI, A.

1998 Société des hommes et société des animaux: F. IMPARATI (a cura di), Studi di Storia e di Filologia Anatolica Dedicati a Giovanni Pugliese Carratelli (Eothen 1), Firenze 1988, pp. 25-37.

BAUTER, J.G. - SAUPPE, H.

1839 Isokratēs, Zurich 1839.

BECCARIA, G.L. (diretto da)

1996 Dizionario di Linguistica Italiana e di Filologia, Metrica, Retorica, Torino 1996.

De Meo, C.

1986 Gli Stili del Latino, Bologna 1986.

FRANCIA, R.

2004 Montagne grandi (e) piccole, (sapete) perchè sono venuto?: Orientalia 73 (2004), pp. 390408.

2013 Storia degli studi sulla poesia ittita e una nuova chiave di lettura di un testo 'classico': CTH 414, Studi Micenei ed Egeo Anatolici (2013).

FRANKFURTER, D

1993 Narrating Power: the Theory and Practice of the Magical Historiola in Ritual Spells, M. MeYer - P. Mirecki (eds.), Ancient Magic and Ritual Power, Leiden - New York - Köln 1993, pp. 457-476.

GoI, S.

$1991 \quad$ Il Segreto delle Filastrocche, Milano 1991.

JAKOBSON, R.

1966 Saggi di linguistica generale, Milano 1966.

KLOEKHORST, A.

2011 Accentuation and Poetic Meter in Hittite: M. Hutter - S. HutTer-Braunsar (eds.), Hethitische Literatur. Überlieferungsprozesse, Textstrukturen, Ausdrucksformen. Akten des Symposiums vom 18. bis 20. Februar 2010 in Bonn, Münster 2011, pp. 157-176.

MELCHERT, H.C.

2007 New Light on the Hittite Verse and Meter: K. JNes-Bley - M.E. Huld - A. Della VolPe M. RobBins DeXter (eds.), Proceedings of the 18th Annual UCLA Indo-European NORDEN, E. Conference. Los Angeles, November 3-4, 2006, Washington 2007, pp. 117-128.

1898 Die antike Kunstprosa vom VI. Jahrhundert V. Chr. bis in die Zeit der Renaissance. Voll. ROVEDA, A. I-II, Stuttgart 1898

2010 Daula daulagna. Filastrocche dell'alta valle dell'Orba, con varianti dell'area ligure e WATKINS, C. piemontese: Quaderni di Semantica XXXI/2 (2010), pp. 239-268.

1995 How to kill a dragon. Aspects of Indo-European poetics, Oxford 1995

WEEDEN, M.

2011 Hittite Logograms and Hittite Scholarship (Studien zu den Böğazköy-Texten 54), Wiesbaden 2011. 